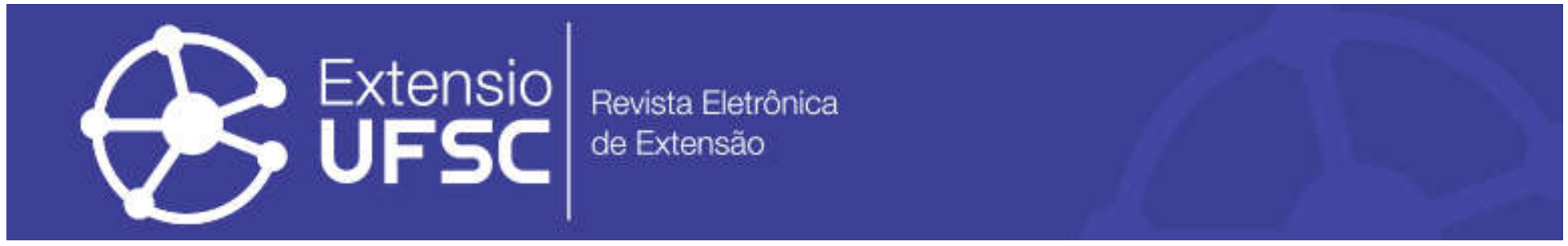

\title{
PROGRAMA DE EDUCAÇÃO TUTORIAL EM ODONTOLOGIA E FONOAUDIOLOGIA DA UNIVERSIDADE FEDERAL DE SANTA CATARINA: UM RELATO DE EXPERIÊNCIA
}

\author{
Lígia Figueiredo Valesan \\ Universidade Federal de Santa Catarina \\ lilivalesan@hotmail.com \\ Gabriella Piazza Maccarini \\ Universidade Federal de Santa Catarina \\ gabipmaccarini@gmail.com \\ Júlia Roberta Régis \\ Universidade Federal de Santa Catarina \\ juliarobertaregis@hotmail.com
}

\begin{abstract}
Murilo Kazuo Iwassake Universidade Federal de Santa Catarina murilo.i.w@gmail.com

Laura Faustino Gonçalves Universidade Federal de Santa Catarina laurafaustinog99@gmail.com

Beatriz Dulcineia Mendes de Souza Universidade Federal de Santa Catarina dentbia@gmail.com
\end{abstract}

\section{Resumo}

O objetivo deste relato de experiência é relatar as atividades e projetos desenvolvidosno Programa de Educação Tutorial de Odontologia e Fonoaudiologia (PET Odonto-Fono) e demonstrar a importância da atuação multidisciplinar na Universidade Federal de Santa Catarina (UFSC). O programaexiste desde 2010 e é formado por discentes e docentes dos cursos de graduação em Odontologia e Fonoaudiologia. O PET Odonto-Fono tem por essência estimular os valores de cidadania e consciência social, bem como elaborar alguns projetos: Capacitações internas, Clínica Integrada Odonto-Fono, PET baseado em Evidências, Projeto Workshop e Palestras, Informa PET, PET Monitoria, PET Tampinhas, Campanha da Cultura Indígena, Planeja PET, PETeca, CinePET, PET Publica e Grupo de Apoio PET. Portanto, o PET OdontoFono promove a transformação social e o enriquecimento da produção científica, além de contribuir naatuação multidisciplinar dos futuros profissionais, capacitando-os e demonstrando a importância doatendimento humanizado e dotrabalho em equipe.

Palavras-chave: Multidisciplinar. Odontologia. Fonoaudiologia.

\section{TUTORIAL EDUCATION PROGRAM IN DENTISTRY AND PHONOAUDIOLOGY AT THE FEDERAL UNIVERSITY OF SANTA CATARINA: AN EXPERIENCE REPORT}

\begin{abstract}
The aim of this experience report is to report the activities and projects developed in Tutorial Education Program for Dentistry and Phonoaudiology (PET Odonto-Fono) and demonstrate the importance of multidisciplinary work at the Federal University of Santa Catarina (UFSC). The program has existed since 2010 and is formed by students and professors of the undergraduate courses in Dentistry and Phonoaudiology. Odonto-Fono PET strives to stimulate the establishment of values reinforce citizenship and social awareness, as well as to complement the curriculum of its participants through its projects: Internal training, Odonto-Fono Integrated Clinic, Evidence based PET, Project Workshop and Lectures, PET reports, PET monitoring, PET campaign, PET planning, PETeca, CinePET, PET publishing and the PET support Group. Therefore, PET Odonto-Fono promotes social transformation and the enrichment of scientific production, in addition to contributing to the multidisciplinary performance of future professionals, training them and demonstrating the importance of humanized care and teamwork.

Keywords: Multidisciplinary. Dentistry. Phonoaudiology.
\end{abstract}


Programa de Educação Tutorial em Odontologia e Fonoaudiologia da Universidade Federal de Santa Catarina: um relato de experiência

\title{
PROGRAMA DE EDUCACIÓN TUTORIAL EN ODONTOLOGÍA Y FONOAUDIOLOGÍA DE LA UNIVERSIDAD FEDERAL DE SANTA CATARINA: UN RELATO DE EXPERIENCIA
}

\begin{abstract}
Resumen
El objetivo de este informe de experiencia es informar las actividades y proyectos desarrollados em el Programa de Educación Tutorial de Odontología y Fonoaudiología (PET Odonto-Fono) y demostrar la importancia de la actuación multidisciplinaria en la Universidad Federal de Santa Catarina (UFSC). El programa existe desde 2010 y consta de estudiantes y profesores de los cursos de graduación en Odontología y Fonoaudiología. El PET Odonto-Fono tiene por esencia estimular la ciudadanía y la conciencia social, así como complementar el currículo de sus participantes con proyectos: Capacitaciones internas, Clínica Integrada Odonto-Fono, PET basado en Evidencias, Taller de proyectos y conferencias, Informa PET, PET Monitoreo, Tapones de botellas de PET, Campaña Cultura indígena, Planes de PET, CinePET, PETeca y el apoyo del público Grupo PET. Por lo tanto, el PET Odonto-Fono promueve la transformación social y el enriquecimiento de la producción científica, además de contribuir al desempeño multidisciplinario de futuros profesionales, capacitándolos y demostrando la importancia del cuidado humanizado y el trabajo en equipo.

Palavras clave: Multidisciplinar. Odontología. Fonoaudiología.
\end{abstract}


Programa de Educação Tutorial em Odontologia e Fonoaudiologia da Universidade Federal de Santa Catarina: um relato de experiência

\section{INTRODUÇÃO}

O Programa de Educação Tutorial (PET) tem como objetivo aprimorar e aperfeiçoar as grades-curriculares dos cursos de ensino superior de acordo com as diretrizes do Ministério da Educação (MINISTÉRIO DA EDUCAÇÃO, 2006). Osdiscentes e docentes participantes atuam na tríade: ensino, pesquisa e extensão, com o intuito de formar cidadãos que saibam trabalhar tanto individualmente quanto em equipes multidisciplinares, de maneira que cada membro desenvolva o seu melhor, contribuindo assim para a formação global do indivíduo (XAVIER; GOULART, 2007).

O projeto foi criado em 1979, pela Coordenação de Aperfeiçoamento de Pessoal de Nível Superior (CAPES), com o nome de "Programa Especial de Treinamento", cuja finalidade era fortalecer o ensino superior no Brasil. Alguns anos depois, em 1999, sua gestão foi assumida pela Secretaria de Educação Superior do Ministério da Educação, e em 2004, passou chamar-se "Programa de Educação Tutorial” (MARTINS, 2007).

Em 2010, a Professora Drª Graziela de Luca Cantofundou,na Universidade Federal de Santa Catarina (UFSC), o PET Odontologia - Fonoaudiologia (PET Odonto-Fono),programa este que tem o propósito de oferecer aos acadêmicos uma visão mais ampla a respeito da relevância da relação multiprofissional. O PET Odonto-Fono tem como base as novas Diretrizes Curriculares Nacionais de 2002 que propuseram uma mudança fundamentadana integralidade da atuação profissional, segundo a qual o profissional deve ser "generalista, humanista, crítico e reflexivo, para atuar em todos os níveis da atenção à saúde, com base no rigor técnico e científico" (Ministério da Educação, 2002).

O sistema estomatognático é uma região anatomofuncional de atuação do cirurgiãodentista e do fonoaudiólogoque compreende estruturas estáticas (mandíbula, maxila, arcos dentários, articulações temporomandibulares e osso hióide) e dinâmicas (músculos mastigatórios, supra e infra-hioideos e de língua, lábios e bochecha) (ANDRADE; CUNHA; REIS, 2017).

O PET Odonto-Fono é um dos poucos programas que conectam dois cursos de graduação na UFSC: Odontologia e Fonoaudiologia.A interação entre os profissionais formados por ambos os cursos se faz necessária para umdiagnóstico mais preciso, prevenção e tratamento das enfermidades relacionadas ao sistema estomatognático (SILVA; CANTO, 2014). De fato, os dois cursos são aliados na promoção da educação em saúde, com maior ênfase na saúde bucal e no desenvolvimento das funções orais (LUCHESI et al., 2016). Essa união de expertises é vantajosa a fim de desenvolver ao longo da vida acadêmica do graduando indivíduos capazes de 
Programa de Educação Tutorial em Odontologia e Fonoaudiologia da Universidade Federal de Santa Catarina: um relato de experiência

trabalhar em equipe, qualificados e humanizados com foco em uma abordagem multidisciplinar, viabilizando o atendimento integral aos pacientes (LUCHESI et al., 2016).

Buscando atuar na tríade ensino, pesquisa e extensão, o PET Odonto-Fono desenvolve projetos que contemplem essas áreas, como as atividades de extensão de cunho social, o desenvolvimento de pesquisas através da prática clínica, capacitações voltadas para o meio acadêmico, além de aulas expositivas que abranjam conteúdos de Fonoaudiologia e Odontologia (MEC, 2006). Dessa forma, o relato de experiência descrito neste artigo tem por objetivo relatar a importância do PET Odonto-Fono na atuação multidisciplinarem saúde, bem como, relatar as atividades e projetos desenvolvidos pelo PET dentro da UFSC.

\section{MATERIAIS E MÉTODOS}

Atualmente, o PET está sob a tutoria da Professora Dra Beatriz Dulcineia Mendes de Souza, responsável pela disciplina de Oclusão do Departamento de Odontologia, e conta com a colaboração voluntária da Professora Dr $^{a}$ Fabiane Miron Stefani, da disciplina de Motricidade Orofacial do Departamento de Fonoaudiologia da UFSC. O time de discentes é formado por9 graduandos do curso de Odontologia e 6 graduandos do curso de Fonoaudiologia, sendo que destes, 12 são bolsistas e 3são voluntários, além da participação voluntária de algumas mestrandas do Programa de Pós-graduação em Odontologia da UFSC.

A produção do conhecimento dentro do PET faz-se por meio de alguns projetos idealizados pelos seus membros, tais como: Capacitações internas, Clínica Integrada OdontoFono, PET baseado em Evidências, PET Publica e o Planeja PET. É importante salientar que os projetos do PET Odonto-Fono procuram garantir aos alunos dos cursos de graduação envolvidos a vivência de experiências extracurriculares, seja de forma direta ou indireta, a fim de enriquecer seus currículos. Outras atividades desenvolvidas tais como o Projeto Workshop e Palestras, Informa PET, “Me formei, e agora?”, PET Monitoria, vêm complementar a formação profissional de todo corpo discente. O PET também possui projetos de extensão eações socioeducativas voltados para a comunidade em geral, são eles: PET Tampinhas, PET Brechó do Desapego, Campanha da Cultura Indígena, Monitoria PET, Grupo de Apoio PET e PETeca.

\section{Projetosdo PET Odonto-Fono:}

Capacitações Internas: compreendem aulas teóricas e práticas com assuntos pertinentes que não são contemplados na grade curricular dos cursos de Fonoaudiologia e Odontologia, 
Programa de Educação Tutorial em Odontologia e Fonoaudiologia da Universidade Federal de Santa Catarina: um relato de experiência

auxiliando na formação diferenciada e generalista voltada ao pensamento crítico dos futuros profissionais.

Informa PET: divulga temas ou datas comemorativas que acontecem no decorrer do ano, por meio das redes sociais como Instagram e Facebook. Atuam como uma mídia de informação semanal e divulgação dos projetos do PET para a comunidade e aos demais alunos da graduação e pós-graduação.

Clínica Integrada Odonto-Fono: tem por objetivo integrar os atendimentos Odontológicos e Fonoaudiológicos que são realizados na ClínicaII da UFSC todas as terças-feiras à tarde. Os pacientes atendidos são da comunidade em geral, encaminhados pela Unidade Básica de Saúde (UBS), ou encaminhados de outras clínicas da universidade de ambos os cursos. Seu objetivo é formar alunos capacitados para o exame, diagnóstico e tratamento de pacientes portadores de disfunções temporomandibulares (DTM), bruxismo e apneia obstrutiva do sono tendo por base a holística multiprofissional (Fotografia 1).

Planeja PET: consiste na discussão de casos clínicos atendidos na Clínica Integrada, com intuito de planejar os casos de forma multidisciplinar, discutir diagnósticos e delinear planos de tratamento, assim como avaliar a evolução de cada paciente com o especialista da área.

PET Tampinhas: tem como objetivo coletar tampinhas de plástico com a finalidade de arrecadarrecursos para a castração de cães e gatos de rua de Florianópolis. As tampinhas são coletadas em caixas distribuídas no Centro de Ciências da Saúde (CCS) da UFSC, e a cada final de mês são recolhidas e distribuídas conforme as cores, para direcionamento à central de coletas das tampinhas.

Projeto Workshop e Palestras: tem por objetivo oferecer à comunidade acadêmica, através de fóruns, seminários, palestras e workshop, habilidades específicas, acerca de diferentes temas que comumente não são abordados na grade curricular da graduação e pós-graduação, nem nos cursos de educação continuada. Os temas abordados são escolhidos pelo grupo conforme a necessidade percebida, ou relatada pelos alunos e professores. No primeiro semestre de 2019 dois workshops, com experts da área, foram realizados abordando os seguintes temas: "Inteligência para a vida: para todos e para cada um" e "A fonte do sucesso está mais perto do que você pensa".

CinePET: consiste na apresentação de um filme, para a comunidade acadêmica, com um assunto pertinente à atualidade, que abordem assuntos voltados à Odontologia e Fonoaudiologia. Além disso, ao final do filme, os professores convidados debatem sobre o tema para suscitar opiniões diversas e o pensamento crítico que possam enriquecer o debate e expor diferentes pontos de vista (Fotografia 2). 
Programa de Educação Tutorial em Odontologia e Fonoaudiologia da Universidade Federal de Santa Catarina: um relato de experiência

Campanha da Cultura Indígena: com o objetivo de valorizar e divulgar a cultura indígena e ainda de auxiliar e apoiar os alunos indígenas presentes na UFSC. A campanha na semana do índio engloba exposições de fotos, divulgações em redes sociais e rodas de conversas com o meio acadêmico e com os alunos membros da Associação dos Estudantes Indígenas da UFSC (AEIUFSC). Além disso, monitorias são realizadas para ajudá-los no melhor aproveitamento acadêmico.

PET Monitoria: tem a intenção de receber e ajudar alunos com dificuldades pedagógicas nas diferentes disciplinas dos cursos de Odontologia e Fonoaudiologia. Além disso, os alunos petianos fornecem orientações e dicas sobre os seus respectivos cursos.

PET Publica: destinado ao desenvolvimento de artigos científicos através de relatos de experiência e casos clínicos. Além disso, incentiva a participação dos integrantes do PET em eventos como encontros acadêmicos, simpósios e congressos para a apresentação e divulgação de trabalhos do grupo (Fotografia 3).

PET Campanha do Desapego: brechó comunitário com duração de uma semana, que será desenvolvido com o intuito de arrecadar fundos para instituições regionais de caridade e para o desenvolvimento de projetos do PET.

PETeca: tem como objetivo disponibilizar livros com temáticas diversas aos acadêmicos com o intuito de ajudar, principalmente, os alunos que sofrem com depressão e ansiedade.

PET Baseado em Evidências: através de aulas expositivas os alunos aprendem a buscar respostas, nas bases de dados LILACS, Pubmed, Cochrane e Science Direct, para responder questões clínicas com base em evidências científicas.

“Me formei e agora?”: esse projeto consiste em palestras, mesas redondas e rodas de conversas com profissionais de ambas as áreas da saúde para disponibilizar, aos alunos das últimas fases dos cursos de Odontologia e Fonoaudiologia, dicas, ideias, relatos de profissionais e incentivos para a vida de recém-formado.

Grupo de Apoio PET: muitos pacientes e alunos sofrem com dores físicas que afetam diretamente sua qualidade de vida. Assim, este projeto tem como objetivo dar apoio a esses pacientes que, em muitos casos, sofrem de depressão, ansiedade, estresse, hipervigilância, dentre outras comorbidades. Com encontros mensais, serão ministradas palestras informativas e diferentes atividades para que esses pacientes encontrem um apoio e amparo no processo de cura ou alívio de suas dores. 
Programa de Educação Tutorial em Odontologia e Fonoaudiologia da Universidade Federal de Santa Catarina: um relato de experiência

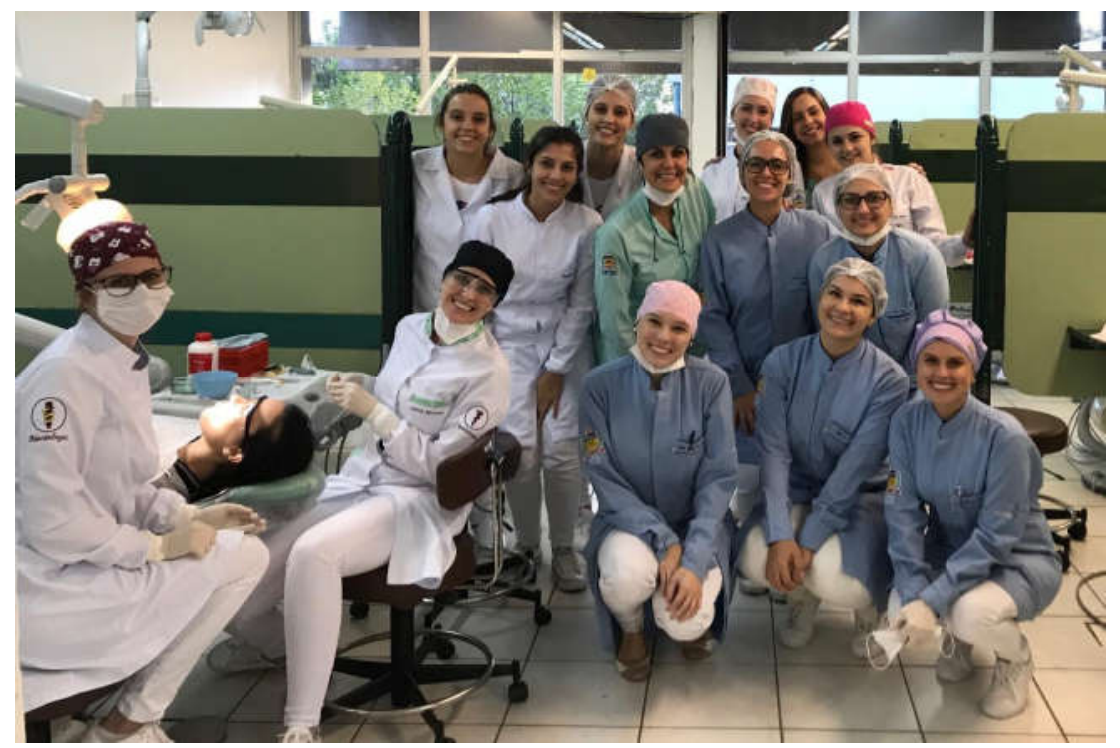

Fotografia 1: Clínica Integrada Odonto-Fono

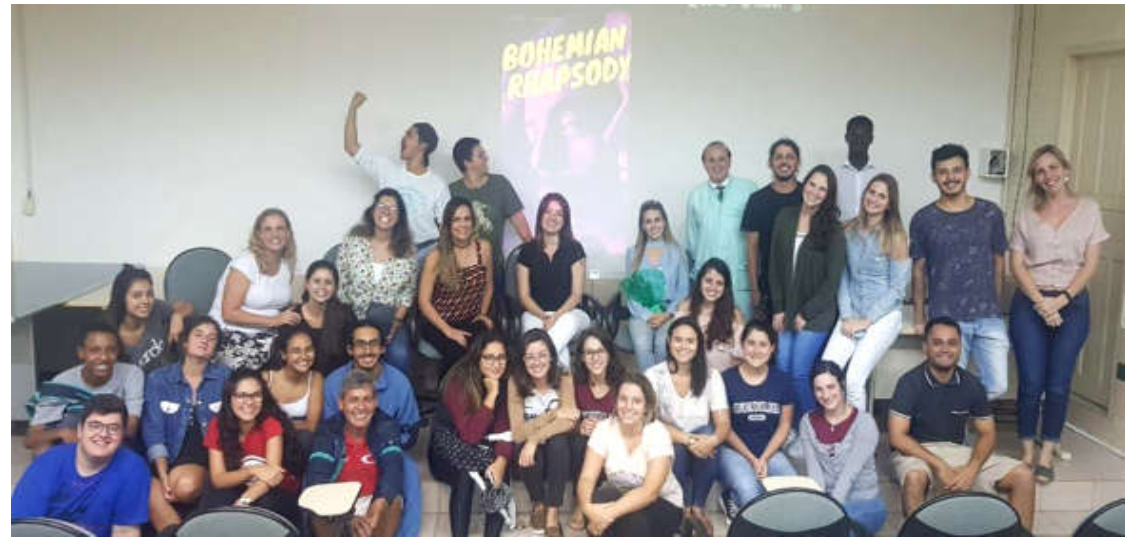

Fotografia 2: CinePET - Filme: Bohemian Rhapsody

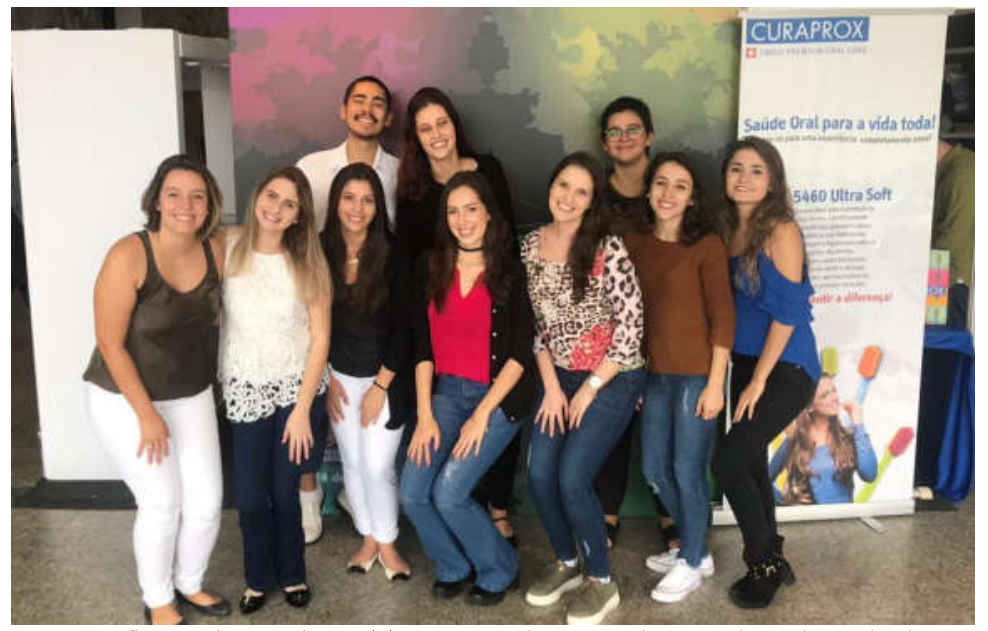

Fotografia 3: Alunos do PET no I Meeting Catarinense de Odontologia 
Programa de Educação Tutorial em Odontologia e Fonoaudiologia da Universidade Federal de Santa Catarina: um relato de experiência

\section{RESULTADOS E ANÁLISES}

Os estudantes universitários devem ter autonomia para buscar conhecimentos que vão além daqueles que o currículo obrigatório tem a oferecer. Para que isso ocorra, é indispensável que os estudantes tenham a possibilidade de cursar disciplinas, fazer estágios e adquirir experiências em áreas do conhecimento diferentes do seu curso de graduação de origem (SILVA; CANTO, 2014). Nesse sentido, o PET Odonto-Fono da UFSC contribui decisivamente para a formação multiprofissional e global do estudante, pois consegue abranger duas áreas aparentemente distintas, mas que se complementam na atenção à saúde do paciente.

A ideia de aprendizado por meio de experiências fora da sala da aula não é nova. Em 2010, Gomes sugeriu a substituição do ensino que se limita à transmissão teórica de conteúdos por um ensino que promova a construção do saber, utilizando de métodos ativos e da análise de situações práticas (GOMES, 2010). Atualmente, esses métodos ativos, ou metodologias ativas, são uma forma alternativa para o processo de ensino-aprendizagem, principalmente nos cursos na área da saúde (PAIVA, 2016). Nesse contexto, os projetos e atividades desenvolvidos pelo PET Odonto-Fono contrapõem-se aos métodos tradicionais aplicados nas salas de aula visto queinstigam o estudantepara que ele seja o protagonista do seu processo de aprendizado, trazendo como consequências oaperfeiçoamento do pensamento crítico e o desenvolvimento de competências para resolução de problemas (TOSTA et al., 2006).

A pluralidade na forma do ensino estimula o desenvolvimento de diferentes habilidades tanto nos alunos quanto nos professores, visto que ao exercer apenas uma forma de trabalho, muitas vezes unidirecional e hierárquica, poucos estudantes são estimulados (BERBEL, 2011). Mitre (2008) relata que a educação deve ser um ato coletivo e de compartilhamento, ou seja, ela não pode ser imposta, mas também não deve ser deixada à própria sorte. Ao desenvolver seus projetos, o PET promove o ensino, de forma variada em sua metodologia, com temas diversos e com olhares voltados aos cursos envolvidos.

A multidisciplinaridade é definida pela integração de várias disciplinas e profissionais que são colocados lado a lado, desenvolvendo iniciativas e garantindo o trânsito entre elas (ROQUETE et al., 2013). A Odontopediatria e a Ortodontia foram as primeiras especialidades que uniram as expertises e, posteriormente, outras especialidades taiscomo prótese, periodontia e oclusão passaram a compreender a importância da troca de conhecimentos e vivências entre os profissionais por meio da interação multidisciplinar (ROQUETE et al., 2013).

No estudo de Mattevi (2011), o autor ressalta a importância do trabalho das equipes de saúde multidisciplinares, as quais desenvolvem ações de promoção e educação em saúde que 
Programa de Educação Tutorial em Odontologia e Fonoaudiologia da Universidade Federal de Santa Catarina: um relato de experiência

resultam na melhoria das condições gerais de saúde da população (MATTEVI, 2011). As duas profissões envolvidas no PET estudam o sistema estomatognático, o que por si só indica que são áreas que se complementam, além de que um desequilíbrio nesse sistema pode promover disfunções neuromusculares, oclusais e articulares (AMARAL et al., 2006) que podem comprometer a qualidade de vida dos indivíduos. Pensando nisso, o PET Odonto-Fono, através da Clínica Integrada Odonto-Fono, enfatiza essa complementaridade e busca a integração de seus estudantesno intuito de tratar estas disfunções e consequentemente melhorar a qualidade de vida dos seus pacientes.

O PET possui noManual de Orientações Básicas, uma carga horária mínima de 20 horas semanais para realização dos projetos de ensino, pesquisa e extensão, as quais devem ser conciliadas com as atividades obrigatórias do currículo, demandando muita organização e integração entre os participantes (DEARO; NAKAYAMA; ROSSIT, 2017). Ao desenvolver atividades da tríade, a universidade proporciona socialização, produção, desconstrução e ressignificação do conhecimento além de se fazer conhecer e produzir como instituição (TAUCHEN, 2009).

Souza e Júnior (2015) relatam em seu estudo que os acadêmicos que participam do PET concluem o curso da graduação em menor tempo do que os que não participam, além de possuírem uma taxa de evasão menor. Com isso, pode-se destacar que a colaboração do PET possibilita um melhor desempenho do universitário durante a graduação, bem como, na sua futura vida profissional (SILVA; CANTO, 2014).

Dentre as principais metas do PET Odonto-Fono, cita-se o engajamento com a transformação de cunho social que pode ser observado pelos projetos: Informa PET, PET Tampinhas, Cine PET, Campanha da Cultura Indígena, PET campanha do Desapego, PETeca, Grupo de Apoio PET e "Me formei e agora?". Portanto, o PET Odonto-Fono procura desenvolver experiências ampliadas que não estão presentes nas estruturas das grades curriculares convencionais, contribuindo para uma formação global e humanística do estudante quanto indivíduo e membro de uma sociedade (TOSTA et al., 2006).

\section{CONSIDERAÇÕES FINAIS}

O PET Odonto-Fono proporciona um enriquecimento da produção científica e atua na transformação social por meio dos seus projetos e atividades. Além disso, traz em sua essência a importância da atuação multidisciplinar e o quanto ela contribui na formação diferenciada dos 
Programa de Educação Tutorial em Odontologia e Fonoaudiologia da Universidade Federal de Santa Catarina: um relato de experiência

indivíduos, formando profissionais capacitados para o trabalho em equipe e, principalmente, para o atendimento humanizado.

\section{REFERÊNCIAS}

AMARAL, E.C. et al. Interrelação entre a Odontologia e a Fonoaudiologia na motricidade orofacial. Revista CEFAC, São Paulo, v.8, n.3, p. 328-36, 2006.

ANDRADE, R. C.; CUNHA, M. D. D.; REIS, A. M. D. C. D. S. Análise morfofuncional do sistema estomatognático em usuários de prótese total convencional do Centro Integrado de Saúde - CIS. Revista CEFAC, São Paulo, v.9, n.5, p, 712-25, 2017.

BERBEL, N. As metodologias ativas e a promoção da autonomia de estudantes. Revista Semina: Ciências Sociais e Humanas, Londrina, v. 32, n. 1, p. 25-40, 2011.

DEARO, P. R.; NAKAYAMA, J. T. O.; ROSSIT, R. A. S. Potencialidades e fragilidades do Programa de Educação Tutorial: percepções de acadêmicos. Caminho Aberto - Revista de Extensão do IFSC, v.4, n.6, p. 37-45, 2017.

GOMES, M. P. C. et al. O uso de metodologias ativas no ensino de graduação nas ciências sociais e da saúde: avaliação dos estudantes. Ciência \& Educação, v. 16, n. 1, p. 181-198, 2010.

LUCHESI, K. F. et al. Fonoaudiologia e Odontologia na Atenção Básica: Relato de Experiência de Educação em Saúde. Distúrbios Comum, São Paulo, v. 28, n. 2, p. 388-93, 2016.

MARTINS, I. L. Educação Tutorial no ensino presencial: uma análise sobre o PET. PETPrograma de Educação Tutorial: estratégia para o desenvolvimento da graduação. Ministério da Educação, Brasília, 2007.

MATTEVI, G. S. et al. A participação do cirurgião-dentista em equipe de saúde multidisciplinar na atenção à saúde da criança no contexto hospitalar. Ciência \& Saúde Coletiva, v. 16, p. 42294236, 2011.

MINISTÉRIO DA EDUCAÇÃO (MEC), Coordenação Geral De Relações Acadêmicas De Graduação Programa De Educação Tutorial - Pet. Manual de Orientações Básicas - PET. p. 1$25,2006$.

MINISTÉRIO DA EDUCAÇÃO (MEC). Conselho Nacional de Educação. Diretrizes Curriculares Nacionais do Curso de Graduação em Odontologia. Diário Oficial da União, Brasília, Seção 1, p. 10, 2002.

MITRE, S. M. et al. Active teaching-learning methodologies in health education: current debates. Ciência \& saúde coletiva, v. 13, p. 2133-2144, 2008.

PAIVA, M. R. F. et al. Metodologias ativas de ensino-aprendizagem: revisão integrativa. SANARE- Revista de Políticas Públicas, v. 15, n. 2, 2016. 
Programa de Educação Tutorial em Odontologia e Fonoaudiologia da Universidade Federal de Santa Catarina: um relato de experiência

ROQUETE, F. F. et al. Multidisciplinaridade, interdisciplinaridade e transdisciplinaridade: em busca de diálogo entre saberes no campo da saúde pública. Revista de Enfermagem do Centro Oeste Mineiro, 2013.

SILVA, T. R.; CANTO, G. L. Integração odontologia-fonoaudiologia: a importância da formação de equipes interdisciplinares. Revista CEFAC, v.16, n.2, p. 598-603, 2014.

SOUZA, R. M.; GOMES J. S. R. Tutorial Education Program: Advances in schooling in physics at Rio Grande do Norte state. Revista Brasileira de Ensino de Física, v. 37, n. 1, p. 1501, 2015.

TAUCHEN, G. et al. O princípio da indissociabilidade universitária: um olhar transdisciplinar nas atividades de ensino, pesquisa e extensão. Porto Alegre, 2009.

TOSTA, R.M. et al. Programa de educação tutorial (PET): uma alternativa para a melhoria da graduação. Psicologia para América Latina, n. 8, p. 0-0, 2006.

XAVIER, B. T. L.; GOULART, D. F. Ensino, pesquisa e extensão consorciados: a fórmula do sucesso do Programa de Educação Tutorial/PET. PETCOM, Bahia, 2007.

Recebido em: 03/07/2019

Aceito em: 24/07/2020 